ON THE DIRECTION-COSINES OF THE AXES OF THE CONICOID, ETC.

\title{
On the Direction-Cosines of the Axes of the Conicoid
}

$$
f(x y z) \equiv a x^{2}+b y^{2}+e z^{2}+2 f y z+2 g z x+26 x y=1 \text {. }
$$

Some time ago I received from $\mathrm{Dr}$ Muirhead the following theorem :- "If $l_{r}, m_{r}, n_{r}(r=1,2, \ldots 3)$ are the direction-cosines of the axes of the conicoid

$$
f(x, y, z)=1, f l_{1} l_{2} l_{3}+g m_{1} m_{2} m_{3}+h n_{1} n_{2} n_{3}=0 . "
$$

In this note a proof and extension of the theorem are given.

The equations for the direction-cosines are

$$
\frac{a l+h m+g n}{l}=\frac{h l+b m+f n}{m}=\frac{g l+f m+c n}{n} .
$$

Therefore

$$
a+\frac{h m_{2}}{l_{2}}+\frac{g n_{2}}{l_{2}}=\frac{h l_{2}}{m_{9}}+b+\frac{f n_{2}}{m_{2}},
$$

and

$$
a+\frac{h m_{3}}{l_{3}}+\frac{g n_{3}}{l_{3}}=\frac{h l_{3}}{m_{3}}+b+\frac{f n_{3}}{m_{3}} .
$$

Subtracting, and remembering that $m_{2} n_{3}-m_{3} n_{2}=l_{1}$, etc., we obtain

$$
\frac{g m_{1}-h n_{1}}{l_{2} l_{3}}=\frac{h n_{1}-f l_{2}}{m_{2} m_{3}}=(\text { similarly }) \frac{f l_{1}-g n_{1}}{n_{2} n_{3}} .
$$

Multiplying numerators and denominators by $f l_{1}, g m_{1}$, and $h n_{1}$ respectively, and adding, we get

$$
f l_{1} l_{2} l_{3}+g m_{1} m_{2} m_{3}+h n_{1} n_{2} n_{3}=0 .
$$

If $D \equiv a b c+2 f g h-a f^{2}-b g^{2}-c h^{2}$ and $A \equiv b c-f^{2}$, etc., we find that each ratio in (1)

$$
=\frac{D l}{A l+B m+G n}=\frac{D m}{H l+B m+F n}=\frac{D n}{G l+F m+C n}
$$

(Geometrically, these follow from the fact that a cone and its reciprocal are coaxal, and (1) gives the direction-cosines of the axes of the cone $f(x, y, z)=0)$.

Therefore as above, we prove

$$
F l_{1} l_{2} l_{3}+G m_{1} m_{2} m_{3}+H n_{1} n_{2} n_{3}=0
$$

From (2) and (3)

$$
\frac{l_{1} l_{2} l_{3}}{g H-h G}=\frac{m_{1} m_{2} m_{3}}{h F-f H}=\frac{n_{1} n_{2} n_{3}}{f G-g F} .
$$

Now if the axes are $O A, O B, O C$, the cone through the coordinate axes and $O A, O B, O C$ is easily seen to be

$$
\frac{l_{1} l_{9} l_{3}}{x}+\frac{m_{1} m_{2} m_{3}}{y}+\frac{n_{1} n_{2} n_{3}}{z}=0 \text {, }
$$


and the cone which touches the planes $B O C, C O A, A O B$ and the coordinate planes is

$$
\sqrt{l_{1} l_{2} l_{3} x}+\sqrt{m_{1} m_{2} m_{3} y}+\sqrt{n_{1} n_{2} n_{3} z}=0 .
$$

Substituting for $l_{1} l_{2} l_{3}, m_{1} m_{2} m_{3}$, and $n_{1} n_{2} n_{3}$, we obtain the equations of these cones.

R. J. T. BELL.

\section{A Method of obtaining Examples on the Multiplication of Determinants.}

In the ordinary text-books on Algebra there is a lack of suitable examples on Multiplication of Determinants. Most of the examples that are given are particular cases of the theorem

in which

$$
D \triangle=D^{n}
$$

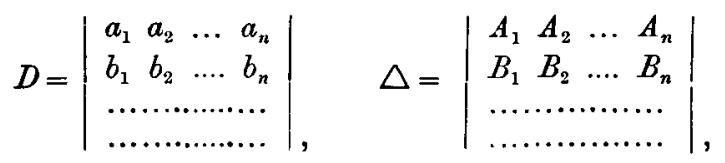

where $A_{1}, A_{2}, \ldots, B_{1}, \ldots$, are the co-factors of $a_{1}, a_{2}, \ldots, b_{1} \ldots$, in $D$.

If the determinant $D$ is chosen at random, in most cases the second determinant $\triangle$ will be too complicated. It is easy, however, to choose $D$ so that factors can be taken out of $\triangle$; and thus a sufficiently simple second determinant is obtained.

For example, let

$$
D=\left|\begin{array}{ccc}
b & a & a \\
a & b & a \\
a & a & b
\end{array}\right|=(2 a+b)(a-b)^{2} .
$$

Then

$$
\triangle=\left|\begin{array}{ccc}
b^{2}-a^{2} & a^{2}-a b & a^{2}-a b \\
a^{2}-a b & b^{2}-a^{2} & a^{2}-a b \\
a^{2}-a b & a^{2}-a b & b^{2}-a^{2}
\end{array}\right| .
$$

Let the factor $b-a$ be taken out of each row of $\triangle$. Then, multiplying the determinant so obtained by $D$, we have

$$
\begin{aligned}
\left|\begin{array}{lll}
b & a & a \\
a & b & a \\
a & a & b
\end{array}\right|\left|\begin{array}{rrr}
a+b & -a & -a \\
-a & a+b & -a \\
-a & -a & a+b
\end{array}\right| & =\left|\begin{array}{ccc}
b^{2}+b a-2 a^{2} & 0 & 0 \\
0 & b^{2}+b a-2 a^{2} & 0 \\
0 & 0 & b^{2}+b a-2 a^{2}
\end{array}\right| \\
& =(b-a)^{3}(b+2 a)^{3} .
\end{aligned}
$$

